J. Dairy Sci. 98:5401-5413

http://dx.doi.org/10.3168/jds.2015-9409

(C) American Dairy Science Association ${ }^{\circledR}, 2015$.

\title{
The effect of calving in the summer on the hepatic transcriptome of Holstein cows during the peripartal period
}

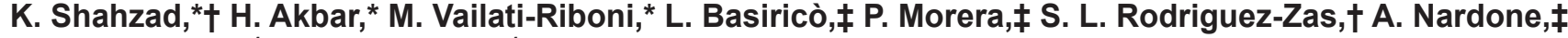 \\ U. Bernabucci, $\ddagger^{1}$ and J. J. Loor* $\dagger^{1}$ \\ *Department of Animal Sciences and Division of Nutritional Sciences, and \\ †lllinois Informatics Institute, University of Illinois, Urbana 61801 \\ łDipartimento di scienze e tecnologie per l'Agricoltura, le Foreste, la Natura e l'Energia (DAFNE), Università degli Studi della Tuscia, \\ Viterbo 01100, Italy
}

\section{ABSTRACT}

The liver is the main metabolic organ coordinating the adaptations that take place during the peripartal period of dairy cows. A successful transition into lactation, rather than management practices alone, depends on environmental factors such as temperature, season of parturition, and photoperiod. Therefore, we analyzed the effect of calving season on the hepatic transcriptome of dairy cows during the transition period. A total of 12 Holstein dairy cows were assigned into 2 groups based on calving season (6 cows March-April, spring; 6 cows June-July, summer, SU). The RNA was extracted from liver samples obtained at $-30,3$, and 35 DIM via percutaneous biopsy and hybridized to the Agilent 44K Bovine (V2) Gene Expression Microarray (Agilent Technologies Inc., Santa Clara, CA). A quantitative PCR on 22 target genes was performed to verify and expand the analyses. A total of 4,307 differentially expressed genes were detected (false discovery rate $\leq 0.05)$ in SU compared with spring. Furthermore, 73 unique differentially expressed genes were detected in SU compared with spring cows after applying a foldchange threshold $\geq 3$ and $\leq-3$. For Kyoto Encyclopedia of Genes and Genomes pathways analysis of differentially expressed genes, we used the dynamic impact approach. Ingenuity Pathway Analysis software was used to analyze upstream transcription regulators and perform gene network analysis. Among metabolic pathways, energy metabolism from lipids, carbohydrates, and amino acids was strongly affected by calving in SU, with a reduced level of fatty acid synthesis, oxidation, re-esterification, and synthesis of lipoproteins, leading to hepatic lipidosis. Glycan-synthesis was downregulated in SU cows probably as a mechanism to counteract

Received February 1, 2015.

Accepted April 25, 2015.

${ }^{1}$ Corresponding authors: jloor@illinois.edu and bernab@unitus.it the progression of this lipidosis. In contrast, calving in the SU resulted in upregulation of gluconeogenesis but also greater use of glucose as an energy source. Among nonmetabolic pathways, the heat-shock response was obviously activated in SU cows but was also associated with inflammatory and intracellular stress response. Furthermore, data support a recent finding that cows experience endoplasmic reticulum stress around parturition. Transcription regulator analysis revealed how metabolic changes are related to important regulatory mechanisms, including epigenetic modification. The holistic analyses of the liver transcriptome response to calving in the summer at high environmental temperatures underscore how transition cows should be carefully managed during this period, as they experience alterations in liver energy metabolism and inflammatory state increasing susceptibility to health disorders in early postpartum.

Key words: heat stress, lactation, parturition, bioinformatics

\section{INTRODUCTION}

During the transition period, several environmental factors including heat stress, photoperiod, and nutritional management affect the health and production efficiency of dairy cows (Dahl et al., 2000; do Amaral et al., 2009). The intensity of heat stress during dry period and lactation can reduce voluntary DMI and negatively affect milk production (do Amaral et al., 2011). Despite the reduced DMI, body fat mobilization does not increase during heat stress, partly due to a reduction in lipolysis and alterations in insulin sensitivity (Baumgard and Rhoads, 2013). The immune system of heat-stressed cows also is compromised (Lacetera et al., 2006) due in part to alterations in gene expression including upregulation of proinflammatory cytokines (Tao et al., 2013). Another well-studied component of cellular responses to heat stress are the heat-shock proteins, which appear to play a major role in eliciting 
immune responses under increased environmental stress in rodents (Campisi et al., 2003) and cows (Catalani et al., 2010).

Although there is some evidence from target-gene analysis that heat stress alters metabolic mRNA expression in liver (do Amaral et al., 2011), it is unknown whether and to what extent other signaling pathways might be affected. For instance, it is well established that the plane of energy nutrition during the dry period can influence the hepatic transcriptome and alter tissue function (Loor et al., 2013; Shahzad et al., 2014). Because of the well-established decrease in DMI induced by heat stress, it is likely that cows calving in the summer (SU) compared with spring (SP) would experience more pronounced decreases in DMI leading to consequent changes in the hepatic transcriptome. Thus, the main objective of this study was to evaluate the effect of calving season on hepatic molecular adaptations in peripartal cows. Furthermore, we sought to use bioinformatics approaches to uncover novel signaling pathways and gene networks that are affected.

\section{MATERIALS AND METHODS}

\section{Experimental Design and Liver Biopsies}

Complete details of the experimental design are available in the Supplemental Materials and Supplemental Table S1 (http://dx.doi.org/10.3168/jds.2015-9409). Briefly, 12 Holstein dairy cows (6 cows March-April, spring, SP; 6 cows June-July, summer, SU) were used for transcriptomics. Those are a subset of 24 Holstein dairy cows, of which 12 calved in spring and 12 calved in the summer. Mean temperature-humidity indices for SP (day: $66.4 \pm 3.8$, night: $56.3 \pm 3.0$ ) and SU (day: $79.5 \pm 2.9$, night: $70.1 \pm 4.7)$ were recorded. At the time of calving, no clinical health problems were observed in cows, and no cow received any treatment for metabolic problems (Basiricò et al., 2011). Liver tissue was harvested via percutaneous biopsy at $-30( \pm 2), 3$, and 35 $\mathrm{d}$ relative to parturition. The biopsies were performed under local anesthesia, and tissue samples were first frozen in liquid nitrogen and then stored at $-80^{\circ} \mathrm{C}$ until real-time PCR and microarray analysis. Blood samples were harvested at $0800 \mathrm{~h}$ from the jugular vein at -30 , -18 , and $-4 \mathrm{~d}$ prepartum (with a final $\pm 2 \mathrm{~d}$ window variation) and 3,17 , and $35 \mathrm{~d}$ postpartum. Milk production was recorded weekly by automatic recorders.

\section{RNA Extraction}

The RNA was extracted from frozen liver tissues using QIAzol Lysis reagent (Qiagen, Chatsworth, CA) and following the manufacturer's protocol. During the procedure, the homogenate was separated into aqueous and organic phases by centrifugation. The RNA was precipitated from the aqueous phase by addition of isopropanol. The isolated RNA was resuspended in DNase-free water and stored at $-80^{\circ} \mathrm{C}$ until quantitative PCR (qPCR) and microarrays. The RNA quality evaluated via RNA integrity number in the Agilent Bioanalyzer (Agilent Technologies Inc., Santa Clara, CA) was greater than 6.5 for all samples.

\section{Microarrays}

cRNA Synthesis, Labeling, and Purification. The microarray experiment was conducted using the 44K-Agilent bovine (V2) gene expression microarray chips (Agilent Technologies Inc.; cat\# G2519F-023647). The methods used for labeling and hybridization were those outlined by Agilent Technologies. Briefly, a total of $200 \mathrm{ng}$ of RNA per sample was used to generate first-strand cDNA, which was subsequently reverse transcribed to cRNA using a low-input quick amp labeling kit (Agilent Technologies Inc.; cat\# 5190-2306). The resulting cRNA was labeled with either $\mathrm{Cy} 3$ or Cy5 fluorescent dye according to the manufacturer's instructions. Purification of the labeled cRNA product was performed with RNeasy mini spin columns (Qiagen, cat\# 74104), and it was subsequently eluted in $30 \mu \mathrm{L}$ of DNase-RNase-free water. The eluted labeled cRNA was quantified in a NanoDrop ND-1000 (Thermo Fisher Scientific Inc., Waltham, MA) to confirm the manufacturer's recommended criteria for yield and specific activity of at least $0.825 \mu \mathrm{g}$ and $\geq 6$.

Fragmentation and Slide Scanning. The labeled cRNA was fragmented and then hybridized to the microarray slide following the manufacturer's protocol. Briefly, $825 \mathrm{ng}$ of $\mathrm{Cy} 3$ and $\mathrm{Cy} 5$ labeled cRNA sample were combined; mixed with $11 \mu \mathrm{L}$ of $10 \mathrm{X}$ Blocking Agent (Agilent Technologies Inc.; cat\# 5188-5281), 2.2 $\mu \mathrm{L}$ of $25 \times$ Fragmentation Buffer (Agilent Technologies Inc.; cat\# 5185-5974), and nuclease-free water (to a final volume of $55 \mu \mathrm{L}$ ); and fragmented at $60^{\circ} \mathrm{C}$ for $30 \mathrm{~s}$. The reaction was then stopped by adding $55 \mu \mathrm{L}$ of $2 \times$ GEx Hybridization Buffer (Agilent Technologies Inc.; cat\# 5190-0403), and the samples were loaded onto the slide. These were hybridized in a rotating hybridization oven at $65^{\circ} \mathrm{C}$ for $17 \mathrm{~h}$. The slides were washed according to the procedures recommended by the manufacturer and scanned using a GenePix 4000B scanner (Axon Instruments Inc., Sunnyvale, CA) and GenePix Pro v.6.1 software. Resulting spots where features were substandard were flagged as bad and excluded from subsequent analysis. 
Table 1. Symbol and name of genes analyzed by qPCR

\begin{tabular}{|c|c|}
\hline Symbol & Gene name \\
\hline$A C O X 1$ & Acyl-coenzyme A oxidase 1, palmitoyl \\
\hline ANGPTL4 & Angiopoietin-like 4 \\
\hline$A P O B$ & Apolipoprotein B \\
\hline CPT1A & Carnitine palmitoyltransferase $1 \mathrm{~A}$ \\
\hline DGAT1 & Diacylglycerol O-acyltransferase 1 \\
\hline EIF2AKЗ & Eukaryotic translation initiation factor $2 \alpha$ kinase 3 \\
\hline FGF21 & Fibroblast growth factor 21 \\
\hline$G P X 1$ & Glutathione peroxidase 1 \\
\hline$H A M P$ & Hepcidin antimicrobial peptide \\
\hline$H P$ & Haptoglobin \\
\hline HSP $70 A 1 A$ & Heat-shock $70 \mathrm{kDa}$ protein $1 \mathrm{~A}$ \\
\hline HSTF1 & Heat-shock transcription factor 1 \\
\hline MTTP & Microsomal triglyceride transfer protein \\
\hline$P C$ & Pyruvate carboxylase \\
\hline PCK1 & Phosphoenolpyruvate carboxykinase 1 \\
\hline $\mathrm{PDK}_{4}$ & Pyruvate dehydrogenase kinase, isozyme 4 \\
\hline PPARA & Peroxisome proliferator activated receptor $\alpha$ \\
\hline$S A A 3$ & Serum amyloid A 3 \\
\hline SOD1 & Superoxide dismutase 1 , soluble \\
\hline SOD2 & Superoxide dismutase 2 , mitochondrial \\
\hline SREBF2 & Sterol regulatory element binding transcription factor \\
\hline$T N F$ & Tumor necrosis factor $\alpha$ \\
\hline
\end{tabular}

\section{Primer Design and Real-Time qPCR}

Protocols for primer design, primer testing, selection of internal control genes for normalization, and other protocols for the qPCR analysis were previously described (Graugnard et al., 2013). Briefly, genes selected as suitable internal control genes based on geNorm analysis included UXT, GAPDH, and RPS9. The geometric mean of these genes was used to normalize gene expression data. The mRNA was used to measure the expression of 22 target genes (Table 1) associated with the heat-shock response (HSP70A1A, HSTF1), fatty acid oxidation (CPT1A, PPARA, ACOX1), hepatokines (FGF21, ANGPTL4), esterification and very-lowdensity lipoprotein assembly (MTTP, APOB, DGAT1, SREBF2), glucose metabolism (PC, PCK1, PDK4), inflammatory mediators (TNF, GPX1, SOD1, SOD2), acute phase protein $(S A A 3, H P, H A M P)$, and endoplasmic reticulum stress (EIF2AK3). Primer sequences have been reported by Graugnard et al. (2013) and Khan et al. (2014).

\section{Statistical Analysis}

Blood metabolites and milk production data were analyzed as repeated measures using the MIXED procedure of SAS (SAS Institute Inc., Cary, NC). The statistical model included as fixed effects time, season, and their interaction. Cow was the random effect. For each analyzed variable, cow was subjected to 3 covariance structures: compound symmetry, autoregressive order one, and unstructured covariance. The covariance structure that had the largest Akaike's information criterion and Schwarz's Bayesian criterion was considered the most desirable for final analysis. For all parameters tested, the best covariance structure was compound symmetry. Results for the main effect of calving season are reported in Table 2.

Statistical analysis of microarrays data was performed using SAS (SAS Institute Inc.). Data from a total of 18 microarrays were adjusted for dye and array effects (Lowess Normalization and array centering). A MIXED model with repeated measures was then fitted to the normalized $\log _{2}$-tranformed adjusted ratios using Proc MIXED. The model included the fixed effects of time $(-30,3$, and $35 \mathrm{~d})$, season (SP and SU), and interaction of time $\times$ season. Cow was considered as uncorrelated random effect. The raw $P$-values were adjusted for the number of genes tested using Benjamini and Hochberg's false discovery rate (FDR; Benjamini and Hochberg, 1995) to account for multiple comparisons. Differences in transcript profiles were considered significant at an FDR-adjusted $P \leq 0.05$. The focus of this manuscript is on the overall differences in liver transcriptome in response to the main effect of group; that is, SU versus SP.

The qPCR data were normalized with the geometric mean of the internal control genes and $\log _{2}$ transformed before statistical analysis. A repeated-measures model was fitted to gene expression data using Proc MIXED

Table 2. Effect of calving in spring (SP) compared with summer (SU) on milk yield and plasma glucose, BHBA, NEFA, urea, and cholesterol

\begin{tabular}{lcccc}
\hline Item & SP & SU & SEM & $P$-value \\
\hline Milk yield $(\mathrm{kg} / \mathrm{d})$ & $33.78^{\mathrm{a}}$ & $23.30^{\mathrm{b}}$ & 2.97 & 0.03 \\
Metabolites (mmol/L) & & & & \\
Glucose & 3.19 & 3.02 & 0.11 & 0.28 \\
Cholesterol & $3.18^{\mathrm{a}}$ & $2.44^{\mathrm{b}}$ & 0.16 & 0.01 \\
NEFA & $0.36^{\mathrm{a}}$ & $0.62^{\mathrm{b}}$ & 0.08 & 0.04 \\
BHBA & 0.57 & 0.52 & 0.07 & 0.60 \\
Urea & $5.28^{\mathrm{a}}$ & $4.54^{\mathrm{b}}$ & 0.21 & 0.03 \\
\hline
\end{tabular}

${ }^{\mathrm{a}, \mathrm{b}}$ Values without a common superscript are significantly different $(P<0.05)$ 
in SAS. The model included the fixed effects of time $(-30,3$, and $35 \mathrm{~d})$, season (SP and SU), and interaction of time $\times$ season. Cow was considered as uncorrelated random effect. An autoregressive covariate structure was used. All means were compared using the PDIFF statement of SAS, and differences were considered statistically significant at $P<0.05$.

\section{Kyoto Encyclopedia of Genes and Genomes Pathways Analysis}

For Kyoto Encyclopedia of Genes and Genomes (KEGG) pathways analysis of differentially expressed genes (DEG) in the comparison of SU versus SP, the dynamic impact approach (DIA) was used. The DIA calculates the overall effect (relevance of a given pathway) and flux (direction of effect), thus allowing evaluation of transcriptome profiles in a more holistic fashion. The detailed methodology of DIA is described elsewhere (Bionaz et al., 2012). Briefly, the whole data set with Entrez gene ID, FDR $\leq 0.05$, fold-change (FC), and $P$-value $\leq 0.05$ was uploaded to DIA. For the analyses a minimum of $30 \%$ annotated genes on the microarray versus the whole genome (Bionaz et al., 2012) was selected. The results of these analyses are reported in figures and tables.

\section{Transcription Regulators and Gene Network Analysis}

Ingenuity pathway analysis (IPA) software (http:/ www.ingenuity.com) was used to analyze the upstream transcription regulators and their connections with other downstream genes that were differentially expressed. For this purpose, a list of DEG along with FC $\geq|2|$ and $P$-values $(\leq 0.05)$ was uploaded into IPA. The results were downloaded and saved for further analysis described below.

\section{RESULTS AND DISCUSSION}

The effects of heat stress and its adverse consequences on DMI, health, milk production, and overall productivity have been widely investigated (St-Pierre et al., 2003; Bernabucci et al., 2010; Liang et al., 2013). Several recent studies have reported target gene expression responses associated with heat stress in mammary, immune cells, and liver (do Amaral et al., 2009, 2011; Tao et al., 2013). Thus, in this study, we have combined target gene expression, whole-transcriptome microarrays, and extensive bioinformatics analyses to study the hepatic response of cows calving in the summer compared with spring. As previously mentioned, this manuscript focuses on the main effect of season (SU vs. SP) during the whole transition period (dry period and early lactation).

When comparing SU versus SP, out of $\sim 44,000$ probes on the microarray, a total of 4,307 (9.79\%) DEG (i.e., 3,820 annotated with Entrez gene ID) were detected at an FDR $\leq 0.05$. After application of a FC threshold, the overall number of DEG was considerably reduced, and only 538 were among those upregulated. It can also be discerned that the number of upregulated genes was higher in both analyses with and without FC threshold. For a clearer picture of DEG during the SU versus $\mathrm{SP}$, we further applied a more stringent criterion of FC threshold at $\geq 3$ and $\leq-3$ and obtained 73 unique genes that were differentially expressed. The results are reported in Tables 3 and 4 . Table 3 contains the genes with positive FC values and Table 4 the genes with negative $\mathrm{FC}$ values.

\section{Expression Patterns of DEG}

The large number of DEG $(3,820)$ underscores the marked effect of calving season on the transcriptome. A total of 2,168 DEG were upregulated and 1,652 downregulated $(P<0.05)$ when cows calved during the SU versus SP. Even after applying a FC criterion of $\geq|2|$ and $|3|$, a similar trend was observed; for example, a total of 531 DEG had a $\geq|2|$ response of which 342 were upregulated and 189 downregulated. These highly affected genes encompass a wide variety of metabolic and nonmetabolic physiological adaptations due to calving season. A similar study dealing with stress response in bovine mammary epithelial cells highlighted the role of several DEG we uncovered (Collier et al., 2006). For example, a number of upregulated DEG in the present study belong to the stress response and repair mechanism, whereas downregulated DEG belong to metabolism and cell cycle. Bioinformatics analyses in the current study allowed for a deeper evaluation encompassing a broader number of biological-molecular pathways (Figure 1).

\section{Summary of the KEGG Pathways}

Among the KEGG categories and subcategories that were enriched with DEG, we focused on 5 main categories (Figure 1). Among these, metabolism, genetic information processing, and cellular processes were overall activated, and environmental information processing and organismal systems were moderately activated (Figure 1). The discussion of transcription profiles will focus on the top 20 most-impacted metabolic and 20 most-impacted nonmetabolic pathways (Figures 2 and 3). 
Table 3. Differentially expressed genes with fold-change $\geq 3$ in liver of cows calving during the summer (SU) compared with spring (SP)

\begin{tabular}{|c|c|c|}
\hline Symbol & Description & SU vs. SP \\
\hline$H P$ & Haptoglobin & 7.44 \\
\hline$G P X 3$ & Glutathione peroxidase 3 (plasma) & 6.85 \\
\hline IL36A & Interleukin $36, \alpha$ & 6.52 \\
\hline MT1E & Metallothionein 1E & 5.03 \\
\hline$A B H D 1$ & Abhydrolase domain containing 1 & 4.69 \\
\hline$D E F B Y$ & Defensin $\beta 7$ & 4.62 \\
\hline$F A M 47 E$ & Family with sequence similarity 47, member $\mathrm{E}$ & 4.59 \\
\hline $\mathrm{EPHX}_{4}$ & Epoxide hydrolase 4 & 4.53 \\
\hline PRSS12 & Protease, serine, 12 (neurotrypsin, motopsin) & 4.30 \\
\hline$D E F B 1$ & Defensin, $\beta 1$ & 4.15 \\
\hline$T B A T A$ & Chromosome 28 open reading frame, human C10orf27 & 4.13 \\
\hline$C A 1$ & Carbonic anhydrase I & 4.08 \\
\hline$M T 2 A$ & Metallothionein 2A & 4.00 \\
\hline TRAFD1 & TRAF-type zinc finger domain containing 1 & 3.98 \\
\hline$M T 1 A$ & Metallothionein-1A & 3.82 \\
\hline$I N M T$ & Indolethylamine N-methyltransferase & 3.79 \\
\hline$D E S$ & Desmin & 3.76 \\
\hline$M I O X$ & Myo-inositol oxygenase & 3.67 \\
\hline $\mathrm{PCP}_{4}$ & Purkinje cell protein 4 & 3.65 \\
\hline FOLR1 & Folate receptor 1 (adult) & 3.64 \\
\hline LOC613867 & Trace amine-associated receptor 7 a-like & 3.63 \\
\hline TMC2 & Transmembrane channel-like 2 & 3.62 \\
\hline$A T P 6 V 1 C 2$ & ATPase, $\mathrm{H}^{+}$transporting, lysosomal $42 \mathrm{kDa}, \mathrm{V} 1$ subunit $\mathrm{C} 2$ & 3.57 \\
\hline$H O P X$ & HOP homeobox & 3.55 \\
\hline $\mathrm{HPS}_{4}$ & Hermansky-Pudlak syndrome 4 & 3.51 \\
\hline FGF21 & Fibroblast growth factor 21 & 3.51 \\
\hline CATHL1 & Cathelicidin 1 & 3.50 \\
\hline JSP.1 & MHC class I JSP.1 & 3.43 \\
\hline$S A A 3$ & Serum amyloid A 3 & 3.43 \\
\hline$N_{T N} 4$ & Netrin 4 & 3.40 \\
\hline$A D C Y r$ & Adenylate cyclase 7 & 3.37 \\
\hline$M U C 15$ & Mucin 15 , cell surface associated & 3.35 \\
\hline$P P L$ & Periplakin & 3.35 \\
\hline ITGA11 & Integrin, $\alpha 11$ & 3.32 \\
\hline C20H5orf49 & Chromosome 20 open reading frame, human C5orf49 & 3.31 \\
\hline$T B C 1 D 14$ & TBC1 domain family, member 14 & 3.30 \\
\hline ISM1 & Isthmin 1 homolog (zebrafish) & 3.29 \\
\hline$D Y X 1 C 1$ & Dyslexia susceptibility 1 candidate 1 & 3.26 \\
\hline LOC517799 & Olfactory receptor, family 52 , subfamily $\mathrm{N}$, member 4-like & 3.21 \\
\hline$H M 13$ & Histocompatibility (minor) 13 & 3.19 \\
\hline$M I C A L 2$ & $\begin{array}{l}\text { Microtubule associated monooxygenase, calponin and LIM domain containing } \\
2\end{array}$ & 3.19 \\
\hline$L B P$ & Lipopolysaccharide binding protein & 3.16 \\
\hline LOC519492 & Olfactory receptor, family 10 , subfamily $\mathrm{T}$, member 2-like & 3.15 \\
\hline GPC3 & Glypican 3 & 3.15 \\
\hline$N W D 1$ & NACHT and WD repeat domain containing 1 & 3.07 \\
\hline PPP1CA & Protein phosphatase 1 , catalytic subunit, $\alpha$ isozyme & 3.03 \\
\hline$A T G_{4} B$ & Autophagy related $4 \mathrm{~B}$, cysteine peptidase & 3.02 \\
\hline LOC509354 & Nucleoside diphosphate kinase 6-like & 3.01 \\
\hline
\end{tabular}

\section{Metabolic Pathways}

Lipid Metabolism. Microarrays along with qPCR results indicate that heat stress during SU led to modification of several lipid-related pathways. Among the most affected pathways, the biosynthesis of fatty acids and unsaturated fatty acids was inhibited in SU compared with SP cows (Figure 2). Use of qPCR also revealed several lipid-synthesis-related genes; for example, SCD, HMGCS1, FADS2, which were downregulated in SU (Figure 4). In ruminant animals, the liver is not a lipogenic organ per se but is the major organ that uptakes NEFA from the blood stream, and their removal from the circulation increases at higher concentrations (Bell, 1979). Thus, the observed downregulation was probably driven by a negative feedback mechanism due to the high level of circulating NEFA (Table 2) in cows calving in SU.

Despite the higher lipomobilization, SU compared with SP cows also had downregulation of expression of genes associated with fatty acid oxidation $(C P T 1 A$, $P P A R A$, and $A C O X 1)$ and esterification and very-low- 
Table 4. Differentially expressed genes with fold change $\leq 3$ in liver of cows calving during the summer (SU) compared with spring (SP)

\begin{tabular}{llc}
\hline Symbol & Description & SU vs. SP \\
\hline BOLA-DQB & Major histocompatibility complex, class II, DQ $\beta$ & -6.77 \\
CHAMP1 & Chromosome alignment maintaining phosphoprotein 1 & -6.48 \\
KIAA0895 & KIAA0855 ortholog & -5.50 \\
AKR1B10 & Aldo-keto reductase family 1, member B10 (aldose reductase) & -5.09 \\
LOC100850628 & Olfactory receptor 51L1-like & -5.06 \\
PVRL1 & Poliovirus receptor-related 1 (herpesvirus entry mediator C) & -4.95 \\
MBOAT2 & Membrane bound O-acyltransferase domain containing 2 & -4.24 \\
MYH7 & Myosin, heavy chain 7, cardiac muscle, $\beta$ & -4.10 \\
ST8SIA1 & ST8 $\alpha$-N-acetyl-neuraminide $\alpha$-2,8-sialyltransferase 1 & -3.91 \\
SCD & Stearoyl-CoA desaturase (delta-9-desaturase) & -3.88 \\
THRSP & Thyroid hormone responsive & -3.79 \\
KCTD20 & Potassium channel tetramerisation domain containing 20 & -3.76 \\
ANKS1B & Ankyrin repeat and sterile $\alpha$ motif domain containing 1B & -3.66 \\
KCNB2 & Potassium voltage-gated channel, Shab-related subfamily, member 2 & -3.47 \\
LRRC49 & Leucine-rich repeat containing 49 & -3.42 \\
RPL4 & Ribosomal protein L4 4.39 \\
LOC100336905 & Uncharacterized LOC100336905 & -3.39 \\
SPAM1 & Sperm adhesion molecule 1 (PH-20 hyaluronidase, zona pellucida binding) & -3.19 \\
PCSK2 & Proprotein convertase subtilisin/kexin type 2 & -3.13 \\
HMGCS1 & HMGCS1 protein-like & -3.11 \\
ASH1L & Ash1 (absent, small, or homeotic)-like (drosophila) & -3.11 \\
FADS2 & Fatty acid desaturase 2 & -3.09 \\
LOC613728 & Nuclear RNA export factor 3-like & -3.09 \\
ACAA1 & Acetyl-CoA acyltransferase 1 & -3.04 \\
KCNJ13 & Potassium inwardly rectifying channel, subfamily J, member 13 & -3.04 \\
\hline
\end{tabular}

density lipoprotein secretion $(A P O B, D G A T 1$, and SREBF2; Figure 4). Thus, the net result of calving during the hot season appears to be an inability of the liver to oxidize the greater load of NEFA, hence, likely rendering cows more susceptible to liver lipidosis. In fact, SU cows had a greater degree of liver triacylglycerol concentration prepartum and early postpartum, with a lower concentration of plasma cholesterol (a lipoprotein marker; Basiricò et al., 2011; Table 2). Overall, these changes indicate that during $\mathrm{SU}$ a greater risk exists for a severe alteration of metabolic status already 4 to $5 \mathrm{wk}$ before calving independently of the adiposity status at calving (Murondoti et al., 2004).

The peroxisome proliferator-activated receptor $\alpha$ $(P P A R A)$ is involved in a variety of cellular functions in the liver of various species (Rakhshandehroo et al., 2010) including ruminants (Bionaz et al., 2013). Thus, at least in monogastrics, PPARA along with its target genes CPT1A, HMGCS1, and ACOX1 play an important role in lipid metabolism. It has been suggested that its function postpartum is increased in transition dairy cows to overcome the negative energy balance (NEB) status (van Dorland et al., 2009; Loor et al., 2013). However, its inhibition during SU compared with SP agrees with qPCR data and liver histology (Basiricò et al., 2011), indicating a lower rate of fatty acid oxidation and metabolism in heat-stressed cows.

Synthesis and degradation of ketone bodies was also overall affected, but a trend of flux toward the positive or negative direction was not evident (Figure 2). This likely indicates an equal response of ketone body synthesis and degradation in both SU and SP. The metabolism of ketone bodies is altered during the transition into lactation (Loor et al., 2007; Osorio et al., 2013) independently of calving season. Most probably, the SP cows experienced the expected physiological increase of ketone body synthesis after calving to overcome the NEB, whereas SU cows had a reduction of this pathway due to the lower oxidation that led to accumulation of fatty acids in liver (Basiricò et al., 2011). In view of the expected adaptations of lipid metabolism pathways after calving, it was surprising to detect an overall induction of fatty acid elongation in mitochondria and fatty acid metabolism pathways in SU compared with SP (Figure 2). Further studies are needed to investigate the role of these pathways in liver during the transition period.

Carbohydrate Metabolism. Among the mostaffected metabolic pathways, the metabolism of propanoate and butanoate (Figure 2) was induced in SU compared with SP cows, indicating a greater degree of glucose metabolism; for example, higher production and utilization. In fact, these results suggest the activation of gluconeogenesis from its precursors (e.g., lactate, glycerol, and alanine; Aschenbach et al., 2010). Using qPCR, it was confirmed that the expression of pyruvate carboxylate $(P C)$ was greater $(P<0.05)$ in SU compared with SP cows (Figure 4), supporting the notion of 


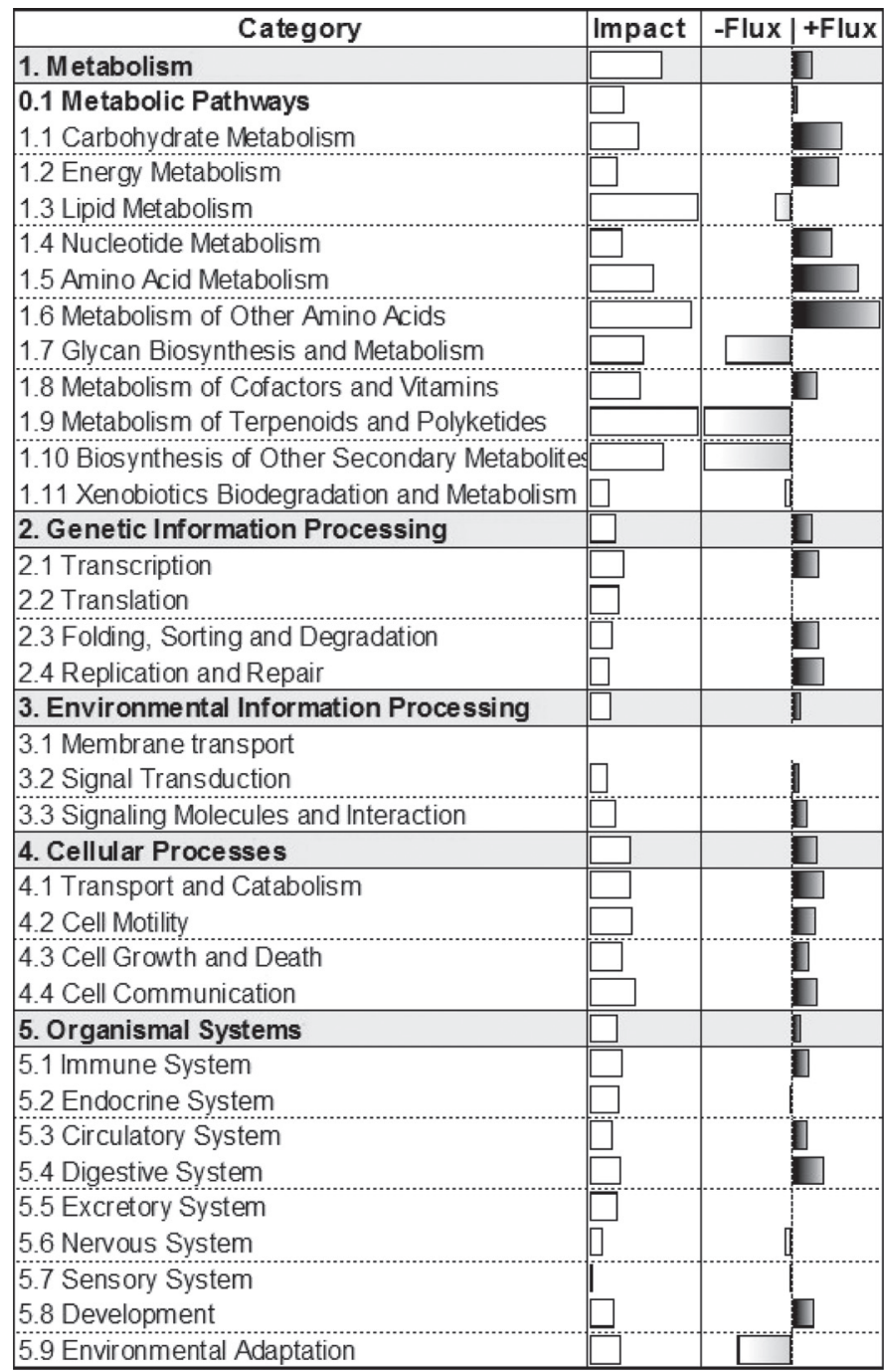

Figure 1. Summary of the Kyoto Encyclopedia of Genes and Genomes categories and subcategories resulting from analysis by dynamic impact approach in liver of cows calving during the summer compared with spring. On the right-hand side, the columns represent the effect (impact) and flux responses. The transparent bars represent the effect values (0 to 100), and the flux columns represent negative $(-)$ and positive $(+)$ flux $(-50$ to +50$)$ based on the direction of the effect. The negative flux is represented with gray bars, and positive flux is represented with black bars.

an increase in glucose synthesis from compounds other than propionate. It was noteworthy that the expression of phosphoenolpyruvate carboxykinase 1 (PCK1) was lower $(P<0.05)$ in the SU compared with SP cows, and that of pyruvate dehydrogenase lipoamide kinase isozyme $4(P D K 4)$ did not differ $(P>0.05)$ between groups. These responses could be related to the lower DMI; that is, less ruminal propionate available, induced by heat stress and potentially greater insulin sensitivity within liver. Such response could have amplified the effect of insulin on the hepatocytes, leading to a reduc-

\begin{tabular}{l}
\hline \multicolumn{1}{|c|}{ Pathways } \\
\hline Fatty acid biosynthesis \\
Fatty acid elongation in mitochondria \\
Terpenoid backbone biosynthesis \\
Synthesis and degradation of ketone bodies \\
Biosynthesis of unsaturated fatty acids \\
Phenylalanine, tyrosine and tryptophan biosynthesis \\
Steroid biosynthesis \\
Propanoate metabolism \\
Fatty acid metabolism \\
Valine, leucine and isoleucine degradation \\
Glutathione metabolism \\
Glycosphingolipid biosynthesis - globo series \\
Tryptophan metabolism \\
Glycosphingolipid biosynthesis - lacto and neolacto series \\
Butanoate metabolism \\
Selenoamino acid metabolism \\
Ubiquinone and other terpenoid-quinone biosynthesis \\
beta-Alanine metabolism \\
Lysine degradation \\
Glycosphingolipid biosynthesis - ganglio series
\end{tabular}

Figure 2. The top 20 most affected metabolic Kyoto Encyclopedia of Genes and Genomes pathways ranked by overall effect value in liver of cows calving during the summer compared with spring. On the right-hand side, the columns represent the effect (impact) and flux responses. The transparent bars represent the effect values $(0$ to $100)$, and the flux columns represent negative $(-)$ and positive $(+)$ flux $(-50$ to +50$)$ based on the direction of the effect. The negative flux is represented with gray bars, and positive flux is represented with black bars.

\begin{tabular}{l}
\multicolumn{1}{|c|}{ Pathways } \\
\hline Antigen processing and presentation \\
PPAR signaling pathway \\
Phagosome \\
Aminoacyl-tRNA biosynthesis \\
Gap junction \\
Carbohydrate digestion and absorption \\
Mineral absorption \\
Cell adhesion molecules (CAMs) \\
RNA polymerase \\
Intestinal immune network for lgA production \\
p53 signaling pathway \\
Complement and coagulation cascades \\
Axon guidance \\
Regulation of actin cytoskeleton \\
Focal adhesion \\
Cytosolic DNA-sensing pathway \\
Regulation of autophagy \\
Notch signaling pathway \\
Tight junction \\
Vasopressin-regulated water reabsorption
\end{tabular}

Figure 3. The top 20 most affected nonmetabolic Kyoto Encyclopedia of Genes and Genomes pathways ranked by overall effect values in liver of cows calving during the summer compared with spring. On the right-hand side, the columns represent the effect (impact) and flux responses. The transparent bars represent the effect values (0 to 100), and the flux columns represent negative $(-)$ and positive $(+)$ flux $(-50$ to +50$)$ based on the direction of the effect. The negative flux is represented with gray bars, and positive flux is represented with black bars. 


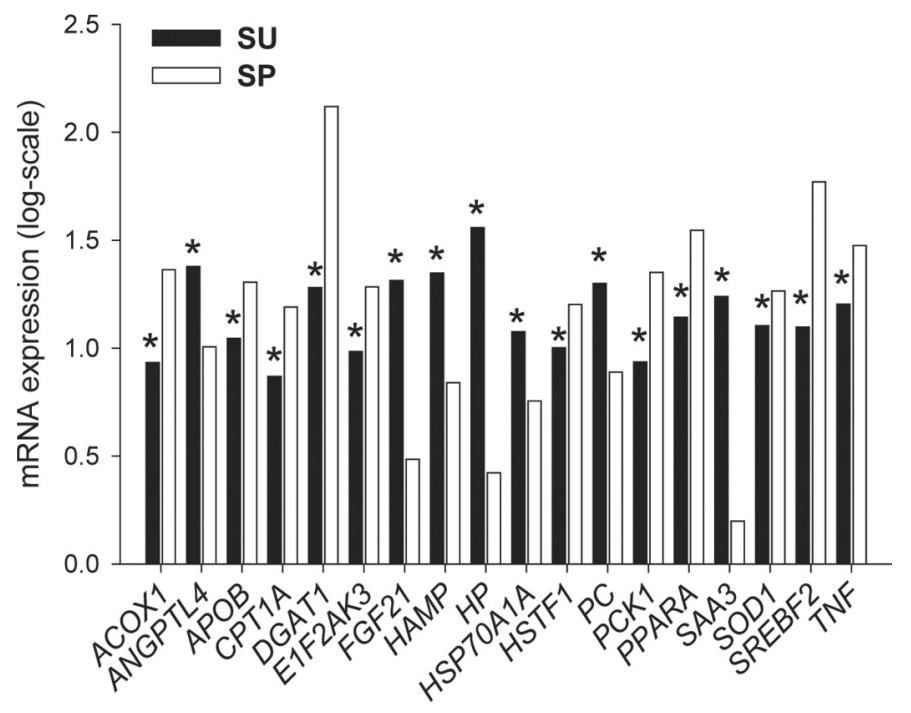

Figure 4. The mRNA expression by quantitative PCR of 18 significantly affected genes tested in liver of cows calving during the summer (SU) compared with spring (SP). ${ }^{*}$ Differs in expression $(P<0.05)$ in SU compared with SP.

tion in oxidation of fatty acids (discussed above) and also allowing for greater tricarboxylic acid cycle flux.

Despite the apparent activation of gluconeogenesis in $\mathrm{SU}$ cows, the microarray and $\mathrm{qPCR}$ results are also suggestive that those cows became more dependent on glucose for energy production (via oxidation) and consequently reduced the amount of glucose available to the mammary gland (Baumgard and Rhoads, 2013). This can be discerned from the lower utilization of mobilized NEFA (discussed above), and the effect and flux of carbohydrate and energy metabolism (Figure 1). This idea agrees with the lower milk production in SU cows, whereas the lower blood glucose concentration postpartum detected in SU compared with SP cows (Basiricò et al., 2011) could be related to lower DMI.

Amino Acid Metabolism. Heat stress seems to have affected the overall metabolism of amino acids in SU cows (Figure 2). The biosynthesis and degradation of several amino acids was activated likely to increase glucose synthesis. In this regard, such response partly explains the activation of biosynthesis of phenylalanine, tyrosine, and tryptophan. Furthermore, the degradation of valine, leucine, isoleucine, and lysine was enhanced. The metabolism of other amino acids, and amino acid-related molecules, was also increased including glutathione, selenoamino acid, and $\beta$-alanine. Among these, glutathione plays an important role in antioxidant-related activities and functions as a regulatory element in disulfide bonds of proteins (Mari et al., 2009). Increased glutathione and antioxidant enzyme synthesis in heat-stressed transition dairy cows has been proposed as an adaptive mechanism to help alleviate oxidative stress (Bernabucci et al., 2002).

The $\beta$-alanine metabolism pathway is linked with other metabolic pathways such as pyrimidine, propanoate, and fatty acid biosynthesis. The ultimate goal of these pathways is to provide nitrogen from skeletal muscle to liver for metabolism; for example, energy synthesis (Timperio et al., 2009). Skeletal muscle catabolism is another recognized mechanism that dairy cows use to provide amino acid carbon to the liver as an energy source during periods of heat stress (Yunianto et al., 1997; Wheelock et al., 2010).

Glycan Biosynthesis and Metabolism. The biosynthesis and metabolism of glycan was also decreased in SU compared with SP cows (Figure 2). Among the top 20 metabolic pathways, all the 3 glycan-related pathways (globo series, ganglio series, and lacto and neolacto series) were inhibited. Glycan plays an important role in a wide range of cellular functions such as protein folding, cell-cell interactions, cellular differentiation, glycosylation, and lysosomal activities (Helenius and Aebi, 2001; Hussain et al., 2012).

Data from mice in which glycan synthesis was suppressed suggest that glycans in hepatocytes are not essential for functions such as sterol, glucose, or lipoprotein metabolism (Jennemann et al., 2010). However, mice fed a high-fat diet treated with an inhibitor of glycan synthesis had a decrease in accumulation of triacylglycerol along with higher concentrations of biomarkers of liver pathology (Zhao et al., 2009). Treatment also reduced the expression of several genes associated with hepatic steatosis, including those involved in lipogenesis, gluconeogenesis, and inflammation. In addition, inhibiting glycan synthesis both prevented the development of steatosis and partially reversed preexisting steatosis (Zhao et al., 2009). Similar effects were reported in another study involving inhibition of glycan synthesis (Bijl et al., 2009). Thus, we hypothesize that the downregulation of the glycan-related pathways in SU cows could be a mechanism to counteract the progression of lipidosis due to the accumulation of lipid in the liver as a consequence of the downregulation of expression of genes involved in fatty acid oxidation and mobilization. Further bovine-specific research is required to verify this hypothesis.

\section{Nonmetabolic Pathways}

Heat Shock and Endoplasmic Reticulum (ER) Stress Response. An increase in temperature can cause protein unfolding and aggregation, which can lead to a variety of cellular pathologies, such as defects of the cytoskeleton, fragmentation of the ER and Golgi 
apparatus, a decrease in number of mitochondria and lysosomes, and poisoning of RNA splicing (Richter et al., 2010). It is commonly believed that the deleterious accumulation of unfolded proteins is the signal starting cellular responses against heat stress, as many morphological and phenotypic effects of heat stress can be explained by the aggregation of proteins and an imbalance of protein homeostasis in general (Richter et al., 2010). The microarray data seem to confirm these mechanisms and highlight the expected activation of the heat-shock response. In fact, different cellular pathways under folding sorting and degradation (i.e., mismatch repair, homologous recombination, base or nucleotide excision repair) and replication and repair were affected by calving season with an overall positive flux and induction in liver of SU compared with SP cows.

The induction of subcategories such as protein processing in endoplasmic reticulum, proteasome, and ubiquitin-mediated proteolysis (Figure 3) strongly support the idea that SU compared with SP cows experienced a greater degree of ER stress. This is also supported by the induction of pathways dealing with glycan synthesis (e.g., O-mannosyl glycan and N-glycan biosynthesis), because a potential role of $\mathrm{N}$-glycans in the liver is to handle the misfolded proteins in the ER during stress conditions. The ER stress and the unfolded protein response (UPR) are closely linked to oxidative stress (Malhotra and Kaufman, 2007). Dairy cows are known to undergo a period of oxidative stress during heat stress (Bernabucci et al., 2002). The simultaneous occurrence of these responses can initiate apoptotic cascades that are deleterious to liver function (Malhotra and Kaufman, 2007).

Analysis by qPCR of 2 key heat-shock proteins (HSP) confirmed that cows in SU compared with SP were heat stressed. The HSP are molecular chaperones, which are induced by the heat-shock response (Feder and Hofmann, 1999) as a mechanism to protect cells against physiological and environmental conditions including heat stress (Wang et al., 2003; Li et al., 2011). The HSP70 protein, encoded by the HSP 70A1A gene, is one that can help protect cells against inflammation, oxidative stress, and heat stress (Morimoto and Santoro, 1998). It is also involved in the replication and repair mechanisms of protein synthesis under ER stress conditions (Wang et al., 2003). The higher expression of $H S P 70 A 1 A$ postpartum (especially at 3 and $35 \mathrm{~d}$ ) in SU compared with SP cows together with microarray results indicates the activation of cytoprotective mechanisms against stress and DNA damage. These mechanisms could also have been induced by the increased energy mobilization (NEFA; Gessner et al., 2014) and by inflammatory and immunological conditions typical of early lactation (Catalani et al., 2010).
Surprisingly, qPCR results revealed that the expression of heat-shock transcription factor 1 (HSF1) was greater in SP compared with SU cows. Heat-shock transcription factor 1 is at the center of the heat-shock response but also can be involved in several cellular regulatory mechanisms such as development, growth, and protein synthesis (Trinklein et al., 2004). Furthermore, the expression of the ER stress sensor protein EIF2AK3 also was greater $(P<0.01)$ in SP compared with SU cows. The EIF2AK3 acts as an ER stress sensor transcription factor, repressing global protein synthesis, whereas spliced-XBP1 is involved in regulation of chaperones and the UPR (Loor, 2010). Along with the DIA indicating an effect and flux toward SU of pathways regarding protein processing and export, these data indicate the possibility that the protein synthesis mechanisms could have been increased by heat stress in SU compared with SP cows (Figure 3) to better cope with the stressful conditions. Further studies are necessary to assess the role of HSF1 and the ER stress in the transition period beside the heat-stress response.

Immune System. The antigen processing and presentation pathway was the most-affected and induced KEGG pathway in SU compared with SP cows. Along with this pathway, phagosome, complement and coagulation cascade, and cytosolic DNA-sensing pathway (Figure 3) is indicative of a more pronounced inflammatory status in liver. The transition period is often characterized by a degree of inflammation (Trevisi et al., 2012) due to the effect of calving itself. However, heat-shock proteins that belong to the Hsp70 family are capable of eliciting an immune response mainly as a result of heat stress to improve the host defense mechanisms (Asea et al., 2000; Campisi et al., 2003). During this response several inflammatory mediators can be activated. Microarray analysis and qPCR data revealed changes in expression of some of these mediators such as tumor necrosis factor $\alpha(T N F)$ and superoxide dismutase 1 (SOD1; Figure 4), or interleukins (IL36A), major histocompatibility complex ( $M H C$ class I), autophagy related 4B $(A T G 4 B)$, and plasma glutathione peroxidase (GPX3; Table 3). The greater expression of these genes indicates a higher inflammatory state during heat stress in $\mathrm{SU}$ cows.

In addition to regulatory elements of the immune system, the overall expression of genes encoding for acute phase proteins (APP), considered reliable biomarkers of inflammation, was higher in SU compared with SP cows (Table 3). Serum amyloid A 3 (SAA3) and haptoglobin $(H P), 2$ of the main APP, had greater $(P<0.01)$ expression in SU cows, together with hepcidin antimicrobial peptide $(H A M P ; P<0.04)$. Serum amyloid $\mathrm{A}$ is immediately secreted from liver after an 
inflammatory response (Faty et al., 2012); that is, it is a major APP that binds to toxins and helps clear lipoproteins out of the liver (Malle et al., 1993). Concentration of HP is also increased due to inflammation in the liver (Shahzad et al., 2014). Similarly, HAMP expression is increased shortly after acute inflammatory stimuli (Pigeon et al., 2001; Nicolas et al., 2002), supporting a role for hepcidin as a positive acute-phase response peptide in dairy cattle.

The inflammatory response has been studied in relation to several lipid- and carbohydrate-related metabolic changes and is known to elicit variation in blood concentrations of cholesterol, NEFA, glucose, and lactate and increase the rate of lipolysis (Hardardottir et al., 1994; Coghe et al., 2000; Kushibiki et al., 2000). Our results agree with these physiological responses underscoring alterations in lipid metabolism, as also detected by blood analysis of the cows used in this study (Table 2) in which NEFA were greater in SU than SP cows.

Hepatokines and the Stress Response. Hepatokines are mainly linked with energy homeostasis and lipid metabolism, and because of the changes we observed in those pathways, it is not surprising that fibroblast growth factor 21 (FGF21) was among the upregulated DEG in SU compared with SP cows (Table 3). Fibroblast growth factor 21 was further measured via qPCR together with another hepatokine, angiopoietinlike 4 (ANGPTL4). Schoenberg et al. (2011) reported an increase of plasma FGF21 after parturition in dairy cows and positively related its concentration, coupled with a greater hepatic mRNA expression, with NEB. Fibroblast growth factor 21 has been identified as an additional hormonal factor involved in the regulation of metabolic adaptations during the transition period (Schlegel et al., 2012). The calving effect on FGF21 expression has been observed in other studies concerning the transition period (Khan et al., 2014; Akbar et al., 2015).

Changes in FGF21 synthesis around parturition could be associated with the increased concentration of growth hormone and glucagon typical of the postpartal period. In nonruminants, both hormones have been recognized as regulators of FGF21 hepatic expression either via direct activation or indirectly via the activation of PPAR $\alpha$ by NEFA (Uebanso et al., 2011). Similar to FGF21, ANGPTL4, another hepatokine controlled by PPAR $\alpha$ (Kersten et al., 2000), is involved in the regulation of energy and lipid metabolism (Hato et al., 2008). Because lipomobilization was greater in SU compared with SP cows (Basiricò et al., 2011; Table 2), their greater expression in the $\mathrm{SU}$ cows underscores the fact that, because of the heat stress, SU cows underwent a greater NEB during the transition period.
These 2 hepatokines, rather than the metabolic adaptation itself, are linked to the stressful conditions of the transition period and the environmental stress of calving during the summer. For instance, the increase during the early postpartal period in dairy cows was linked with the ER stress phenomena; that is, ER stress induces a greater hepatic expression of FGF21 (Gessner et al., 2014). An intramammary LPS challenge with LPS in early-postpartum cows resulted in marked upregulation of ANGPTL4 in liver tissue (Graugnard et al., 2013), confirming that it may function as an acute-phase response protein. As proposed by Akbar et al. (2015), our data agree with a possible role of circulating ANGPTL4 and FGF21 as APP reflecting metabolic stress. Their application as biomarkers during the peripartal period should undergo further investigation.

Transcription Regulators. To understand the transcription mechanisms occurring within the liver during spring and summer, we studied the role of transcription regulators and their connections with other downstream genes. The IPA analysis using genes with FC $\geq 2$ resulted in a total of 29 transcription regulators identified. The upstream transcription regulator network is shown in Supplemental Figure S1 (http:// dx.doi.org/10.3168/jds.2015-9409), and more detailed information is reported in Table 5 . The network highlighted 4 transcription regulators (HDAC5, IRF3, USF2, and XBP1), all of which were upregulated in SU compared with SP cows. These could be considered central for the modulation of the hepatic transcriptome around calving during heat stress.

The affected genes (Table 5) were mostly related to energy metabolism, with a large portion being related to lipid anabolic and catabolic pathways (i.e., $A C A D M$, $H A D H A, C P T 1 B$, and FASN). Except USF2, all regulators were involved in the modulation of genes related to ER stress, UPR, and inflammation response (Table 5). It is well established that XBP1 is a central regulator of the UPR (Lee et al., 2003). Several target genes related to cell division or apoptosis were overall upregulated by the transcription regulators (Table 5). Recent data suggested the involvement of HDAC5 in hepatic regeneration (Huang and Rudnick, 2014). As heat stress could lead to cell damage, and many HSP have antiapoptotic functions (Kregel, 2002), the fact that this particular regulator was among the most affected suggests that it can elicit a protective role during the heat-shock response. Overall, the IPA network analysis underscores the importance of lipid metabolism together with the effect on hepatocyte health during the transition period in heat-stressed cows.

As judged by the number of target genes (Table 5), a noteworthy output generated from the IPA analysis 
Table 5. Upstream differentially expressed transcription regulators and their target genes with fold change $\geq|3|$ in liver of cows calving during the summer (SU) compared with spring (SP)

\begin{tabular}{|c|c|}
\hline $\begin{array}{l}\text { Transcription } \\
\text { regulator }\end{array}$ & Target gene and response in SU vs. SP \\
\hline$D N A J B 6$ & 个KRT8, PHKG2, ${ }^{1} \uparrow K R T 18$ \\
\hline ELF3 & $\uparrow P R K C S H, \uparrow K R T 18$ \\
\hline ID1 & $\uparrow C T G F, \uparrow C D C 20, \uparrow C D K 4$ \\
\hline IRF3 & $\downarrow P P P 2 R 3 A, \downarrow I F N W 1$, IFNA16, ${ }^{1} \uparrow U S P 18$ \\
\hline PPP1R27 & $\uparrow P P P 1 C A$ \\
\hline$S N W 1$ & $S I R T 7, \uparrow T U B B, \uparrow E E F 1 A 1, \uparrow E E F 1 G$ \\
\hline SRSF2 & $\uparrow S N C A, \uparrow S I R T r$ \\
\hline USF2 & $\uparrow U C P 2, \uparrow K A R S, \uparrow S 100 A 9, \downarrow F A S N, \uparrow C P T 1 B, \uparrow C D K 4, \downarrow T H R S P$ \\
\hline$X B P 1$ & $\downarrow F A S N, \uparrow F K B P 11, \uparrow H M 13$ \\
\hline
\end{tabular}

${ }^{1}$ Included in the Ingenuity Pathway Analysis network but without any up- or downstream regulation.

was the seemingly important role of HDAC5 in coordinating hepatic response to heat stress. The protein encoded by this gene belongs to a histone deacetylase (HDAC), a group of enzymes that are part of the epigenetic mechanism of transcription regulation. Histone deacetylase can cleave an acetyl group from a histone, resulting in a more compact DNA helix that prevents access to genetic information by the cell transcription machinery (de Ruijter et al., 2003). The end result of deacetylation is downregulation or silencing of the expression of genes located in the given DNA region. Data from Table 5 indicate that upregulation of $H D C A 5$ was linked with both downregulation and upregulation of target genes. The fact that most of its target genes were upregulated in SU cows leads us to speculate that some transcription inhibitor located in the acetylated histone area was silenced, hence, minimally affecting expression and upregulation of the target genes. It is also likely that the genes regulated by HDAC5 are subject to control from other transcription regulators; for example, SIRT'7 is controlled by MAGED1, NOCL2, SNW1, and SRSF2 (Table 5).

\section{CONCLUSIONS}

Management of the transition cow is already at the center of the modern dairy industry. The hepatic transcriptome of transition dairy cows is strongly affected by season of calving. Besides expanding our knowledge of molecular adaptations coordinating production responses (e.g., milk production, energy balance), results from this study underscore how environmental stress not only alters energy metabolism in liver, but also induces an inflammatory and intracellular stress response. When combined with the physiological inflammatory state characteristic of the peripartal period, the overall effect is a greater susceptibility to health disorders early postpartum. The holistic analyses of the liver transcriptome response to calving in the summer at high environmental temperatures supports recent findings that cows experience ER stress around parturition. Furthermore, novel mechanisms related to epigenetic regulation of DNA transcription appear to be involved in the response to extreme environmental conditions. Their functional relevance merits further study.

\section{ACKNOWLEDGMENTS}

The study was supported in part by a grant from the Italian Government (MiPAAF-MICROCLILAT, D.M. 6738/22-03-2012, and MiPAAF-INNOVAGEN, D.M. 10750/7303/2011, projects) and by Hatch funds under project ILLU-538-914 (National Institute of Food and Agriculture, Washington, DC).

\section{REFERENCES}

Akbar, H., T. M. Grala, M. Vailati Riboni, F. C. Cardoso, G. Verkerk, J. McGowan, K. Macdonald, J. Webster, K. Schutz, S. Meier, L. Matthews, J. R. Roche, and J. J. Loor. 2015. Body condition score at calving affects systemic and hepatic transcriptome indicators of inflammation and nutrient metabolism in grazing dairy cows. J. Dairy Sci. 98:1019-1032.

Aschenbach, J. R., N. B. Kristensen, S. S. Donkin, H. M. Hammon, and G. B. Penner. 2010. Gluconeogenesis in dairy cows: The secret of making sweet milk from sour dough. IUBMB Life 62:869-877.

Asea, A., S. K. Kraeft, E. A. Kurt-Jones, M. A. Stevenson, L. B. Chen, R. W. Finberg, G. C. Koo, and S. K. Calderwood. 2000. HSP70 stimulates cytokine production through a CD14-dependant pathway, demonstrating its dual role as a chaperone and cytokine. Nat. Med. 6:435-442.

Basiricò, P. M., N. Lacetera, B. Ronchi, A. Nardone, and U. Bernabucci. 2011. Down-regulation of hepatic ApoB100 expression during hot season in transition dairy cows. Livest. Sci. 137:49-57.

Baumgard, L. H., and R. P. Rhoads. 2013. Effects of heat stress on postabsorptive metabolism and energetics. Annu. Rev. Anim. Biosci. 1:311-337. 
Bell, A. W. 1979. Lipid metabolism in the liver and selected tissues and in the whole body of ruminant animals. Prog. Lipid Res. $18: 117-164$.

Benjamini, Y., and Y. Hochberg. 1995. Controlling the false discovery rate-A practical and powerful approach to multiple testing. J. Roy. Stat. Soc. B Met. 57:289-300.

Bernabucci, U., N. Lacetera, L. H. Baumgard, R. P. Rhoads, B. Ronchi, and A. Nardone. 2010. Metabolic and hormonal acclimation to heat stress in domesticated ruminants. Animal 4:1167-1183.

Bernabucci, U., B. Ronchi, N. Lacetera, and A. Nardone. 2002. Markers of oxidative status in plasma and erythrocytes of transition dairy cows during hot season. J. Dairy Sci. 85:2173-2179.

Bijl, N., M. Sokolović, C. Vrins, M. Langeveld, P. D. Moerland, R. Ottenhoff, C. P. A. A. van Roomen, N. Claessen, R. G. Boot, J. Aten, A. K. Groen, J. M. F. G. Aerts, and M. van Eijk. 2009. Modulation of glycosphingolipid metabolism significantly improves hepatic insulin sensitivity and reverses hepatic steatosis in mice. Hepatology 50:1431-1441.

Bionaz, M., S. Chen, M. J. Khan, and J. J. Loor. 2013. Functional role of PPARs in ruminants: Potential targets for fine-tuning metabolism during growth and lactation. PPAR Res. 2013:1-28.

Bionaz, M., K. Periasamy, S. L. Rodriguez-Zas, W. L. Hurley, and J. J. Loor. 2012. A novel dynamic impact approach (DIA) for functional analysis of time-course omics studies: Validation using the bovine mammary transcriptome. PLoS ONE 7:e32455.

Campisi, J., T. H. Leem, and M. Fleshner. 2003. Stress-induced extracellular Hsp72 is a functionally significant danger signal to the immune system. Cell Stress Chaperones 8:272-286.

Catalani, E., M. Amadori, A. Vitali, U. Bernabucci, A. Nardone, and N. Lacetera. 2010. The Hsp72 response in peri-parturient dairy cows: Relationships with metabolic and immunological parameters. Cell Stress Chaperones 15:781-790.

Coghe, J., C. H. Uystepruyst, F. Bureau, J. Detilleux, T. Art, and P. Lekeux. 2000. Validation and prognostic value of plasma lactate measurement in bovine respiratory disease. Vet. J. 160:139-146.

Collier, R. J., C. M. Stiening, B. C. Pollard, M. J. VanBaale, L. H. Baumgard, P. C. Gentry, and P. M. Coussens. 2006. Use of gene expression microarrays for evaluating environmental stress tolerance at the cellular level in cattle. J. Anim. Sci. 84(Suppl.):E1E13.

Dahl, G. E., B. A. Buchanan, and H. A. Tucker. 2000. Photoperiodic effects on dairy cattle: A review. J. Dairy Sci. 83:885-893.

de Ruijter, A. J., A. H. van Gennip, H. N. Caron, S. Kemp, and A. B. van Kuilenburg. 2003. Histone deacetylases (HDACs): Characterization of the classical HDAC family. Biochem. J. 370:737-749.

do Amaral, B. C., E. E. Connor, S. Tao, J. Hayen, J. Bubolz, and G. E. Dahl. 2009. Heat-stress abatement during the dry period: Does cooling improve transition into lactation? J. Dairy Sci. 92:59885999

do Amaral, B. C., E. E. Connor, S. Tao, M. J. Hayen, J. W. Bubolz, and G. E. Dahl. 2011. Heat stress abatement during the dry period influences metabolic gene expression and improves immune status in the transition period of dairy cows. J. Dairy Sci. 94:86-96.

Faty, A., P. Ferre, and S. Commans. 2012. The acute phase protein serum amyloid A induces lipolysis and inflammation in human adipocytes through distinct pathways. PLoS ONE 7:e34031.

Feder, M. E., and G. E. Hofmann. 1999. Heat-shock proteins, molecular chaperones, and the stress response: Evolutionary and ecological physiology. Annu. Rev. Physiol. 61:243-282.

Gessner, D. K., G. Schlegel, R. Ringseis, F. J. Schwarz, and K. Eder. 2014. Up-regulation of endoplasmic reticulum stress induced genes of the unfolded protein response in the liver of periparturient dairy cows. BMC Vet. Res. 10:46.

Graugnard, D. E., K. M. Moyes, E. Trevisi, M. J. Khan, D. Keisler, J. K. Drackley, G. Bertoni, and J. J. Loor. 2013. Liver lipid content and inflammometabolic indices in peripartal dairy cows are altered in response to prepartal energy intake and postpartal intramammary inflammatory challenge. J. Dairy Sci. 96:918-935.

Hardardottir, I., C. Grunfeld, and K. R. Feingold. 1994. Effects of endotoxin and cytokines on lipid metabolism. Curr. Opin. Lipidol. $5: 207-215$
Hato, T., M. Tabata, and Y. Oike. 2008. The role of angiopoietinlike proteins in angiogenesis and metabolism. Trends Cardiovasc. Med. 18:6-14.

Helenius, A., and M. Aebi. 2001. Intracellular functions of N-linked glycans. Science 291:2364-2369

Huang, J., and D. A. Rudnick. 2014. Elucidating the metabolic regulation of liver regeneration. Am. J. Pathol. 184:309-321.

Hussain, M. R. M., M. Hassan, I. Afzal, and A. Afzal. 2012. Role of Gal and GalNAc containing glycans in various physiological processes. Egypt. J. Med. Hum. Genet. 13:1-9.

Jennemann, R., U. Rothermel, S. Wang, R. Sandhoff, S. Kaden, R. Out, T. J. van Berkel, M. Aerts, K. Ghauharali, C. Sticht, and H. j. Gröne. 2010. Hepatic glycosphingolipid deficiency and liver function in mice. Hepatology 51:1799-1809.

Kersten, S., S. Mandard, N. S. Tan, P. Escher, D. Metzger, P. Chambon, F. J. Gonzalez, B. Desvergne, and W. Wahli. 2000. Characterization of the fasting-induced adipose factor FIAF, a novel peroxisome proliferator-activated receptor target gene. J. Biol. Chem. 275:28488-28493.

Khan, M. J., C. B. Jacometo, D, E. Graugnard, M. N. Correa, E. Schmitt, F. Cardoso, and J. J. Loor. 2014. Overfeeding dairy cattle during late-pregnancy alters hepatic PPARalpha-regulated pathways including hepatokines: Impact on metabolism and peripheral insulin sensitivity. Gene Regul. Syst. Bio. 8:97-111.

Kregel, K. C. 2002. Heat shock proteins: Modifying factors in physiological stress responses and acquired thermotolerance. J. Appl Physiol. 92:2177-2186.

Kushibiki, S., K. Hodate, Y. Ueda, H. Shingu, Y. Mori, T. Itoh, and Y. Yokomizo. 2000. Administration of recombinant bovine tumor necrosis factor-alpha affects intermediary metabolism and insulin and growth hormone secretion in dairy heifers. J. Anim. Sci. $78: 2164-2171$.

Lacetera, N., U. Bernabucci, D. Scalia, L. Basiricò, P. Morera, and A. Nardone. 2006. Heat stress elicits different responses in peripheral blood mononuclear cells from Brown Swiss and Holstein cows. J. Dairy Sci. 89:4606-4612.

Lee, A. H., N. N. Iwakoshi, and L. H. Glimcher. 2003. XBP-1 regulates a subset of endoplasmic reticulum resident chaperone genes in the unfolded protein response. Mol. Cell. Biol. 23:7448-7459.

Li, Q., J. Han, F. Du, Z. Ju, J. Huang, J. Wang, R. Li, C. Wang, and J. Zhong. 2011. Novel SNPs in HSP70A1A gene and the association of polymorphisms with thermo tolerance traits and tissue specific expression in Chinese Holstein cattle. Mol. Biol. Rep. 38:2657-2663.

Liang, D., C. L. Wood, K. J. McQuerry, D. L. Ray, J. D. Clark, and J. M. Bewley. 2013. Influence of breed, milk production, season, and ambient temperature on dairy cow reticulorumen temperature. J. Dairy Sci. 96:5072-5081.

Loor, J. J. 2010. Genomics of metabolic adaptations in the peripartal cow. Animal 4:1110-1139.

Loor, J. J., M. Bionaz, and J. K. Drackley. 2013. Systems physiology in dairy cattle: Nutritional genomics and beyond. Annu. Rev. Anim. Biosci. 1:365-392.

Loor, J. J., R. E. Everts, M. Bionaz, H. M. Dann, D. E. Morin, R. Oliveira, S. L. Rodriguez-Zas, J. K. Drackley, and H. A. Lewin. 2007. Nutrition-induced ketosis alters metabolic and signaling gene networks in liver of periparturient dairy cows. Physiol. Genomics 32:105-116.

Malhotra, J. D., and R. J. Kaufman. 2007. Endoplasmic reticulum stress and oxidative stress: A vicious cycle or a double-edged sword? Antioxid. Redox Signal. 9:2277-2293.

Malle, E., A. Steinmetz, and J. G. Raynes. 1993. Serum amyloid A (SAA): An acute phase protein and apolipoprotein. Atherosclerosis 102:131-146.

Mari, M., A. Morales, A. Colell, C. Garcia-Ruiz, and J. C. FernandezCheca. 2009. Mitochondrial glutathione, a key survival antioxidant. Antioxid. Redox Signal. 11:2685-2700.

Morimoto, R. I., and M. G. Santoro. 1998. Stress-inducible responses and heat shock proteins: New pharmacologic targets for cytoprotection. Nat. Biotechnol. 16:833-838. 
Murondoti, A., R. Jorritsma, A. C. Beynen, T. Wensing, and M. J. Geelen. 2004. Unrestricted feed intake during the dry period impairs the postpartum oxidation and synthesis of fatty acids in the liver of dairy cows. J. Dairy Sci. 87:672-679.

Nicolas, G., C. Chauvet, L. Viatte, J. L. Danan, X. Bigard, I. Devaux, C. Beaumont, A. Kahn, and S. Vaulont. 2002. The gene encoding the iron regulatory peptide hepcidin is regulated by anemia, hypoxia, and inflammation. J. Clin. Invest. 110:1037-1044.

Osorio, J. S., P. Ji, J. K. Drackley, D. Luchini, and J. J. Loor. 2013. Supplemental Smartamine M or MetaSmart during the transition period benefits postpartal cow performance and blood neutrophil function. J. Dairy Sci. 96:6248-6263.

Pigeon, C., G. Ilyin, B. Courselaud, P. Leroyer, B. Turlin, P. Brissot, and O. Loréal. 2001. A new mouse liver-specific gene, encoding a protein homologous to human antimicrobial peptide hepcidin, is overexpressed during iron overload. J. Biol. Chem. 276:78117819 .

Rakhshandehroo, M., B. Knoch, M. Muller, and S. Kersten. 2010. Peroxisome proliferator-activated receptor alpha target genes. PPAR Res. 2010:612089.

Richter, K., M. Haslbeck, and J. Buchner. 2010. The heat shock response: Life on the verge of death. Mol. Cell 40:253-266.

Schlegel, G., J. Keller, F. Hirche, S. Geissler, F. J. Schwarz, R. Ringseis, G. I. Stangl, and K. Eder. 2012. Expression of genes involved in hepatic carnitine synthesis and uptake in dairy cows in the transition period and at different stages of lactation. BMC Vet. Res. 8:28.

Schoenberg, K. M., S. L. Giesy, K. J. Harvatine, M. R. Waldron, C. Cheng, A. Kharitonenkov, and Y. R. Boisclair. 2011. Plasma FGF21 is elevated by the intense lipid mobilization of lactation. Endocrinology 152:4652-4661.

Shahzad, K., M. Bionaz, E. Trevisi, G. Bertoni, S. L. Rodriguez-Zas, and J. J. Loor. 2014. Integrative analyses of hepatic differentially expressed genes and blood biomarkers during the peripartal period between dairy cows overfed or restricted-fed energy prepartum. PLoS ONE 9:e99757.

St-Pierre, N. R., B. Cobanov, and G. Schnitkey. 2003. Economic losses from heat stress by US livestock industries. J. Dairy Sci. 86(Suppl.):E52-E77.

Tao, S., E. E. Connor, J. W. Bubolz, I. M. Thompson, B. C. do Amaral, M. J. Hayen, and G. E. Dahl. 2013. Short communication:
Effect of heat stress during the dry period on gene expression of mammary tissue and peripheral blood mononuclear cells. J. Dairy Sci. 96:378-383.

Timperio, A. M., A. D'Alessandro, L. Pariset, G. M. D'Amici, A. Valentini, and L. Zolla. 2009. Comparative proteomics and transcriptomics analyses of livers from two different Bos taurus breeds: "Chianina and Holstein Friesian. J. Proteomics 73:309-322.

Trevisi, E., M. Amadori, S. Cogrossi, E. Razzuoli, and G. Bertoni. 2012. Metabolic stress and inflammatory response in high-yielding, periparturient dairy cows. Res. Vet. Sci. 93:695-704.

Trinklein, N. D., J. I. Murray, S. J. Hartman, D. Botstein, and R. M. Myers. 2004. The role of heat shock transcription factor 1 in the genome-wide regulation of the mammalian heat shock response. Mol. Biol. Cell 15:1254-1261.

Uebanso, T., Y. Taketani, H. Yamamoto, K. Amo, H. Ominami, H. Arai, Y. Takei, M. Masuda, A. Tanimura, N. Harada, H. Yamanaka-Okumura, and E. Takeda. 2011. Paradoxical regulation of human FGF21 by both fasting and feeding signals: Is FGF21 a nutritional adaptation factor? PLoS ONE 6:e22976.

van Dorland, H. A. S. Richter, I. Morel, M. G. Doherr, N. Castro, and R. M. Bruckmaier. 2009. Variation in hepatic regulation of metabolism during the dry period and in early lactation in dairy cows. J. Dairy Sci. 92:1924-1940.

Wang, S., K. R. Diller, and S. J. Aggarwal. 2003. Kinetics study of endogenous heat shock protein 70 expression. J. Biomech. Eng. 125:794-797.

Wheelock, J. B., R. P. Rhoads, M. J. Vanbaale, S. R. Sanders, and L. H. Baumgard. 2010. Effects of heat stress on energetic metabolism in lactating Holstein cows. J. Dairy Sci. 93:644-655.

Yunianto, V. D., K. Hayashi, S. Kaneda, A. Ohtsuka, and Y. Tomita. 1997. Effect of environmental temperature on muscle protein turnover and heat production in tube-fed broiler chickens. Br. J. Nutr. 77:897-909.

Zhao, H., M. Przybylska, I. H. Wu, J. Zhang, P. Maniatis, J. Pacheco, P. Piepenhagen, D. Copeland, C. Arbeeny, J. A. Shayman, J. M. Aerts, C. Jiang, S. H. Cheng, and N. S. Yew. 2009. Inhibiting glycosphingolipid synthesis ameliorates hepatic steatosis in obese mice. Hepatology 50:85-93. 